\title{
Management of Demented Patients in Emergency Department
}

\author{
Lavinia Valeriani \\ Casa di Cura San Raffaele Nomentana, Via Emilio Praga, 39, 00137 Rome, Italy \\ Correspondence should be addressed to Lavinia Valeriani, lav75@libero.it
}

Received 27 December 2010; Accepted 5 February 2011

Academic Editor: Fabrizio Vecchio

Copyright (C 2011 Lavinia Valeriani. This is an open access article distributed under the Creative Commons Attribution License, which permits unrestricted use, distribution, and reproduction in any medium, provided the original work is properly cited.

\begin{abstract}
The hospitalization of the elderly with acute illness is one of the most discussed in the organization of health services, it is not yet clear whether the hospital is really the best response to the needs of the elderly, especially those with cognitive impairment. Despite evidence of possible adverse effects of hospitalization (immobilization, acute confusional state resulting in sedation, risk of falls, intestinal sub-ileus), there has been an increasing use of the hospital, particularly to specialist services. Regardless of the benefits from the shelter (instrumental diagnosis and prompt treatment of acute somatic disease), in people with dementia it needs to identify the characteristics of the person (cognitive impairment, functional status, somatic comorbidity, social and familial status), the personal needs and, therefore, diagnostic and therapeutic targets which must be assumed for that sick person during hospitalization. To this end, it is fundamental the role of assessment and diagnostic orientation that takes place in the Department of Emergency and Acceptance (DEA), which mainly receives patients at the hospital. Even before the hospital recovery it is therefore essential to check how many elderly patients with cognitive impairment that belong to the DEA, and what are their needs.
\end{abstract}

\section{Epidemiological Data}

Over the past 10 years an increasing proportion of elderly people, especially those with dementia, had recourse to the Department of Emergency and Acceptance (DEA): the elderly, in respect to young and adult people, on average, have more urgent requests, stay longer in the department (both for diagnosis and for therapy), use more resources and staff time [1]. Despite a great number of tests and procedures, diagnoses tend to be less accurate: this condition is explained by the atypical presentation of many diseases, the clinical and pharmacological comorbidity, which tend to complicate the presentation, the diagnosis, and the treatment of acute or chronic disease. In addition, older people often belong to the DEA for diseases that require intensive care: a study carried out for 5 years in 352 hospitals in the USA and England, it was found that the percentage of admission in polyfunctional intensive therapy (PIT) of patients over 65 years is very high (above 50\%). In the total group of patients belonging to the DEA, the subsequent admission to the PIT was mainly for medical conditions (53\% in England, $63 \%$ in USA), and less for acute surgical diseases (48 vesus $41 \%$, resp.) [2]. The data shows that elderly patients who belong to the DEA, and among these also patients suffer from dementia, are presented to the hospital for somatic diseases or acute and severe surgical procedures that require often intensive treatment. It should be noted, moreover, a different intensively diagnostic/therapeutic attitude between different countries: USA PIT hospitalization of elderly patients from the DEA has increased, especially for acute somatic diseases. This reflects in part the difference in prevalence in the elderly population, and probably represents a different attitude in the decisions of hospitalization of elderly patients between the two countries. A study conducted in Italy showed that $21 \%$ of people who belong to the DEA are over 65 years. The percentage of those who are hospitalized increased with age: it goes from $11 \%$ of those under 65 years, to $56 \%$ of the over nineties. The majority of patients hospitalized in medical departments from DEA is over sixty-five (60\%), but represents only $25 \%$ in surgical wards. Among all patients hospitalized and then hospitalized for the DEA, 6\% suffer from severe dementia. The sick elderly patient who suffers from dementia that reaches the DEA, therefore, is often hospitalized because of the seriousness of his condition requires acute somatic hospital treatment. Moreover, because of the greater disease severity, the duration of hospital stay 
in patients over eighty years is longer than the hospital stay of younger females (7.9 versus 5.8, resp., days for males, 6.8 versus 4.1 in women). An increase in patients, over 85 years, who belong to the DEA: $71 \%$ versus $65 \%$ of the general population. $71 \%$ versus $65 \%$ of the general population. Among these patients, more than $80 \%$ are suffering from dementia, of which $30 \%$ suffer from severe dementia, and $20 \%$ from the moderate form of disease (patients followed at home by a caregiver). Another interesting fact that emerges from the study is the cause of hospitalization: $43 \%$ of the patients are evaluated for acute somatic pathology, and $33 \%$ for chronic heart failure. The percentage increases for each age group; over-85-year group always (including the dementia for $80 \%$ ) that have the highest percentage (41\%) of hospitalization for acute somatic illness. The recent studies paint a scene so disturbing: a high number of elderly patients and, among these, a high percentage of people with dementia, belong to a structure for acute, historically more prepared to manage acuities in young and adult. The future scenario must necessarily change, since the number of patients with these characteristics tends to increase over time [1]. Among the possible explanations for this phenomenon, apart from the increase in the average age of the population and worsening of chronic somatic illnesses and comorbidity (especially in people with dementia) in general, it increases the vision of the DEA as a replacement more quickly and technological than the caregivers, and the lack of an adequate social/assistance support at home [3].

\section{The Main Pathological Conditions}

The majority of patients with dementia pertains to the DEA for an acute somatic or surgical illness, or a chronic heart failure. The diseases that most often drive the elderly to apply for an urgent evaluation are cardiovascular diseases (angina, heart failure, arrhythmias, and syncope) or respiratory diseases (acute exacerbation of chronic bronchitis, bronchial asthma, and pneumonia), in addition, cancer (cancer of the lung, breast, and large bowel), and neurological diseases (acute cerebrovascular disease, altered state of consciousness) [4]. Among surgical emergencies, the most common diagnosis is related to trauma and fractures, caused mainly by falling to the ground. Other diseases that result in the arrival at the DEA are clinical emergencies that require different level of intervention: sometimes less technological and more clinical (dehydration, urinary tract infections, intestinal subileus, delirium, behavioral disturbances, and subsequent guidance of therapeutic prescription) others more specific to the setting of care (acute respiratory failure from respiratory infection, acute myocardial infarction, and sepsis). Finally, in some cases, the patient with dementia is sent to the DEA for clinical problems related to an incorrect home management: oversedation from psychopharmacological treatment, side effects from medications (iatrogenic hypotension, hypoglycemia jatrogena). See Table 1 for a summary.

In these situations a careful medical and medication history is the necessary instrument (low-tech, but related to the practice of good clinical practice) that would lead to the diagnosis and solution of the problem. It should also be considered that there is a different prevalence of somatic diseases in different stages of Alzheimer's disease and other dementias. Those that are usually associated with mild to moderate dementia are the tumours, diabetes, gastrointestinal disease, while those associated with severe dementia are pneumonia and other infectious diseases, stroke, malnutrition, hip fractures, bed sores [5]. See Table 2 for a summarization.

In this case, dementia complicates the management of chronic diseases, compromising the ability of patients to detect the presence of an incipient fault, to measure the severity of the disease, to accurately report symptoms, and to follow closely the prescriptions [6].

\section{The Evaluation of the Patient in the Emergency Department}

A difficult problem in clinical management and in supporting people with dementia is the impairment in the ability to report somatic symptoms: this ability seems directly related to insight of disease, different from person to person. Patients with dementia generally tend to subreport symptoms of organic disease, and therefore may be at increased risk of somatic disease: a result could be that many potentially treatable medical conditions are overlooked. Furthermore, the clinical manifestations of somatic diseases may be atypical in patients with dementia, the onset of acute illness or exacerbation of persistent disease may occur, rather than with classic signs and symptoms, with confusion: hyperkinetic or hypokinetic delirium, the second even more difficult to detect and interpret. Finally, somatic diseases may occur with sudden onset of behavioral problems, or a modification if in their background: usually there is an increase in frequency and severity of BPSD (behavioural and psichological symptoms of dementia), such as agitation, insomnia, busy, deliria, or hallucinations. A study showed that there is a clear difference between painful somatic symptoms reported spontaneously by the person with dementia, compared to those required by an observer [7]. Considering both the symptoms reported spontaneously and those evoked after request, patients with MCI and very mild complained significantly more symptoms than cognitively intact patients. The number of painful symptoms changed in patients suffering from dementia of varying severity (from very mild to severe): a greater severity of cognitive impairment corresponded to a lower number of somatic symptoms reported. Since the diagnostic orientation in medicine is mainly based on reported symptoms, it is possible that many diseases, potentially treatable, may not be diagnosed in the person with cognitive impairment. This condition is central in the clinical treatment of organic disease in patients with dementia, because the nonresponse (and the consequent failure to treat) of somatic disease significantly affects both somatic health and evolution of cognition and functional status [8].

\section{Clinical Assessment}

The assessment of somatic diseases in the course of dementia is a phase of the multidimensional assessment, which often 
TABLE 1: The diseases that most often drive the elderly to apply for an urgent evaluation.

\begin{tabular}{l} 
Medical emergency \\
Cardiovascular diseases (angina, heart failure, arrhythmias, syncope) \\
Respiratory (acute exacerbation of chronic bronchitis, bronchial asthma, pneumonia) \\
Cancer (cancer of the lung, breast, large bowel) \\
Neurological diseases (acute cerebrovascular disease, altered state of consciousness) \\
\hline Chirurgical emergency \\
Trauma and fractures \\
Clinical emergency \\
Dehydration, urinary tract infections, intestinal sub-ileus, delirium, behavioral disturbances and subsequent guidance of therapeutic \\
prescription \\
Acute respiratory failure from respiratory infection, acute myocardial infarction, sepsis \\
Clinical problems related to an incorrect home management \\
Oversedation from psychopharmacological treatment, side effects from medications (iatrogenic hypotension, hypoglycemia jatrogena) \\
\hline
\end{tabular}

TABLE 2: Principal pathologies associated with patients with dementia.

\author{
For mild to moderate dementia: \\ Tumours, diabetes, gastrointestinal disease \\ For severe dementia: \\ Pneumonia and other infectious diseases, stroke, malnutrition, hip fractures, bed sores
}

suffers from the problems associated with comorbidity, or that is, entirely neglected. One of the targets of care of patients with dementia is in fact the prevention of complications (infection, malnutrition, incontinence, or delirium) which result in an increased risk of hospitalization and increased mortality in short and medium term [6]. A careful management of possible comorbidity could therefore slow the functional decline and limit the complications. In patients with dementia, even in the early stages of the disease, medical history should be collected or at least confirmed by the principal caregiver or by a person who knows the personal history. The doctor involved in the evaluation of the patient should be able to detect the presence of signs and somatic symptoms in the acute phase.

The risk that you may incur, then, rather than an overestimation of pain in patients with dementia is the underestimation of the symptom in older cognitively compromised patients. The question is to understand how it is possible to detect somatic symptoms in patients who have communication problems and memory disturbances. It is evident that, while for chronic symptoms we must rely on memory and observation of the caregiver, for the detection of acute pain you can take advantage of signs that may accompany the symptoms and be directly observed. Firstly, a sudden change in cognitive status of a patient is always an alarm bell. When family members report a rapid deterioration, or an unusual confusion, the possibility that the patient has pain should always be investigated. We must observe the gestures and movements, asking the patient to report the discomfort. The same process must be implemented when the patient experiences a sudden agitation, insomnia, or when they show unusual apathy and drowsiness. Determining the amount of pain is much more difficult. The correct attitude is to try any way because of the pain and to treat both the disease and the symptom. The risk of a too conservative practice is to leave the most compromised patients-those who cannot communicate their pain in any way-alone with their pain. In addition to symptom assessment, the assessment of somatic health of the patient with dementia is based on the concepts already defined by the multidimensional geriatric assessment associated with the clinical evaluation of the patient. In the person with dementia, the examination is of particular importance in order to capture significant signs, that are the indicator of an underlying organic disease not reported or underestimated by the patient and the family. Similarly, given the difficulty of communication of the patient, the clinical signs should be carefully considered: the difficulty breathing or tachypnea is a sign of an underlying respiratory or cardiac disease, regardless of the reported symptom (dyspnea). Finally, incorrect posture could be due to side effects of a prolonged neuroleptic treatment, and antalgic attitudes give an indication related to the districts affected by pain. Furthermore, the presentation of somatic or acute surgical diseases can be atypical, for example, pneumonia rarely occurs with fever, chest pain, and cough, but simply with a catastrophic effect on functional status, an event that represents the most significant clinical manifestation. In front of nonspecific and atypical symptoms and signs of acute somatic illness (lethargy, delirium, and rapid functional decline), it is important that the evaluation of patients with dementia, which refers to the DEA, is the most complete and accurate. In DEA the beats are tight, and there is often a need to evaluate many patients quickly, with the risk of neglecting the details: for this reason it is possible that the atypical signs of the diseases of the patient with dementia are neglected, resulting in delay on diagnosis and treatment of disease. It is therefore necessary, 
TABLe 3: Clinical assessment of patients with dementia.

Anamnesis: medical history collected or at least confirmed by the principal caregiver or a person who knows the history

Risk of underestimation of the symptom in older cognitively compromised

Objective examination: patient visit in order to capture significant clinical signs

Useful indicator of an underlying organic disease not reported or underestimated by the patient and the family

Pharmacological anamnesis: drug history of the patient

Many drugs may cause side effects, especially when administered by not clinical prepared persons

Vital signs: for better understanding of the patient's general condition

Determination of blood pressure, heart rate, oxygen saturation (blood gas, or), body temperature, and glycemia

with complex patients, to retrieve that good clinical practice that researches all possible causes, starting with a thorough medical history. Events interpreted as normal for adults are not normal for the elderly suffering from dementia the recent onset of fever, decreased hydration, push to try different pathologies with a patient who does not communicate and which shows only a drowsiness. Still, the drug history is important, especially if we think that many drugs (e.g., oral hypoglycemic agents, antihypertensives, neuroleptics) may cause side effects, especially when administered by persons not prepared by the clinical point of view (family members). The assessment of vital signs, finally, is essential for a better understanding of the patient's general condition: the determination of blood pressure, heart frequency, oxygen saturation (or blood gas), body temperature, and glycemia. On the basis of the geriatric history and the clinical evaluation, it becomes easier to guide implementation of blood chemistry and instrumental analysis in DEA while avoiding unnecessary and expensive tests, both in terms of patient suffering (execution time, discomfort for the patient and their families) that in economical terms. The evaluation of the patient with dementia which refers to the DEA, therefore, requires more attention than the adult, as it needs a specific and careful approach, which considers every possible cause of somatic or surgical illness to achieve a good diagnostic orientation, a prerequisite for the selection of the correct treatment regimen. Table 3 reports the principal clinical assessments of patients with dementia.

\section{The Path in DEA}

When patients with dementia belong to the DEA for somatic problems or the exacerbation of cognitive or behavioral disorders, the key problem is to understand the real necessity and usefulness of hospitalization; that is, if the issue in which the patient with dementia is presented to the DEA is solved in the same emergency room, or if it becomes necessary hospitalization for diagnosis and treatment. In fact, in some cases nonspecific symptoms (confusion, agitation, anxiety, or, conversely, apathy, and drowsiness) may represent the onset of an acute somatic disease (lung infection, acute heart failure, acute myocardial infarction, and fractures), which requires correct hospitalization. In this case, the diagnostic, clinical and instrumental capacity of the staff of the DEA would lead to a disease orientation that should be treated in a hospital. Sometimes, on the contrary, from the evaluation of the DEA, medical conditions requiring hospitalization emerge: it is often the patient's family that led him to the DEA, frightened by a series of nonspecific symptoms that cannot be understoond (conduct disorder, bone and joint pains, abdominal pain from chronic constipation, and dyspepsia). The minor clinical problems can be addressed directly in the DEA, for which the patient can be treated on site (pain therapy, oral antibiotics, and enema evacuees) and be returned to their home, or in nursing homes of origin. This attitude has a twofold advantage: firstly it solves the problem directly to the patient and his family members, reducing the discomfort and inconvenience of a hospitalization. On the other hand, it allows the patient to return to their place of residence, thus avoiding the risk of delirium, which is high for the hospitalized patient with dementia. Certain negative conditions must be, however, remember: even today, too often, the patient suffering from dementia is an uncomfortable patient. When the patient arrives at the DEA, the doctor knows that, most likely, he will tract a number of problems that characterize a complex patient. Often the risk is to reduce the attention, to make a superficial assessment, assuming a series of chronic pathologies, and therefore not worthy of consideration. Superficiality and carelessness, unfortunately, do not allow an overall assessment of the patient, with the risk of failing the expectations of the patient and family members, or to neglect the real problem of the patient (e.g., to deal with the agitated patient with sedatives without thinking about the cause that could be pneumonia). Finally, particular attention should be paid to the environment: the DEA is often a noisy, crowded place, where all patients are understandably suffering from an illness, seeking an answer quickly, not including delays or procedures, also because few medics find the time to stop and explain it. Stress related to pain, noise, and unfamiliar environment are reflected even more heavily on the patient suffering from dementia, who find themselves in a "tower of Babel" where just few stop talk to them, trying to understand their problems and to cheer them up. Little attention is given to their needs, including physiological ones: if they have to wait in the DEA for several hours before the visit, it is difficult for them to ask for help, as they suffer from dementia, they do not pay attention to their needs: hydration, using the bathroom, eating, and caring for their somatic pain. The risk is to accentuate the symptoms, including behavioural ones, and arrive at the time of the visit with a patient even more confusing and therefore, in our 
eyes, even more incomprehensible. The future of the DEA, if it is to become efficient and effective, is to specialize in these patients, who will increasingly need quick and competent responses [9].

\section{Conclusions}

A large number of people who come to the DEA suffer from a severe form of dementia: $12 \%$ of patients who are admitted to medical ward from the emergency department suffer from severe dementia, so it is possible to envisage a double rate if we include patients with dementia in mild to moderate impairment. The hospital and the staff of the DEA, in general, are not prepared to deal with the continuing (and increasing gradually over time) requirement of care by people with dementia, regardless of its severity. In fact, with increasing age, it increases the clinical complexity of patients that belong to the hospital. It is necessary that the staff of the DEA is prepared (and not just on the field) to the assessment and planning of the elderly patient with dementia: how to recognize cognitive, sensory deficits, to identify the patient's functional status and social resources at home are fundamental factors that drive both the diagnostic orientation and treatment choices (hospital care versus at home care). The risk you take is to use the outdated diseaseoriented method that does not help the understanding of geriatric pathologies. As noted in a recent study, most physicians working in the DEA in the USA reported higher levels of anxiety as a result of the large number of elderly patients who belong to the hospital, and their inability to cope with the complexity of the patient. The low level of experience, and the lack of specific training in geriatric medicine for acute care and in relation to the elderly and their families, are factors contributing to increased stress for staff [9]. The future scenario should include a plan to improve the quality of care, through a specific theoretical and practical training for all personnel working in an emergency ward: the objective is to train skilled professionals to handle the urgency of the young and adult, but also specialized in the treatment of acute diseases of the elderly patients with dementia [10].

\section{References}

[1] F. Aminzadeh and W. B. Dalziel, "Older adults in the emergency department: a systematic review of patterns of use, adverse outcomes, and effectiveness of interventions," Annals of Emergency Medicine, vol. 39, no. 3, pp. 238-247, 2002.

[2] H. Wunsch, D. Harrsion, W. Linde-Zwirble, D Angus, and K. Rowan, "Differences in ICU admissions for the elderly between the United States and the United Kingdom," Critical Care, vol. 10, supplement 1, p. 429, 2006.

[3] P. T. Donnan, D. W. T. Dorward, B. Mutch, and A. D. Morris, "Development and validation of a model for predicting emergency admissions over the next year (PEONY): a UK historical cohort study," Archives of Internal Medicine, vol. 168, no. 13, pp. 1416-1422, 2008.

[4] P. Ray, S. Birolleau, Y. Lefort et al., "Acute respiratory failure in the elderly: etiology, emergency diagnosis and prognosis," Critical Care, vol. 10, no. 3, article no. R82, 2006.
[5] D. W. K. Kay, T. A. Holding, B. Jones, and S. Littler, "Dependency in old age: a comparison of mental and physical factors," International Journal of Geriatric Psychiatry, vol. 6, no. 12, pp. 833-844, 1991.

[6] L. Teri, S. M. McCurry, S. D. Edland, W. A. Kukull, and E. B. Larson, "Cognitive decline in Alzheimer's disease: a longitudinal investigation of risk factors for accelerated decline," The Journals of Gerontology Series A, vol. 50, no. 1, pp. M49-M55, 1995.

[7] G. B. Frisoni, V. Fedi, C. Geroldi, and M. Trabucchi, "Cognition and the perception of physical symptoms in the community-dwelling elderly," Behavioral Medicine, vol. 25, no. 1, pp. 5-12, 1999.

[8] A. Burns, R. Jacoby, P. Luthert, and R. Levy, "Cause of death in Alzheimer's disease," Age and Ageing, vol. 19, no. 5, pp. 341344, 1990.

[9] J. G. Schumacher, G. T. Deimling, S. Meldon, and B. Woolard, "Older adults in the emergency department: predicting physicians' burden levels," Journal of Emergency Medicine, vol. 30, no. 4, pp. 455-460, 2006.

[10] J. McCusker, M. Cole, M. Abrahamowicz, L. Han, J. E. Podoba, and L. Ramman-Haddad, "Environmental risk factors for delirium in hospitalized older people," Journal of the American Geriatrics Society, vol. 49, no. 10, pp. 1327-1334, 2001. 


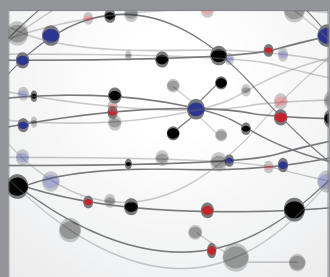

The Scientific World Journal
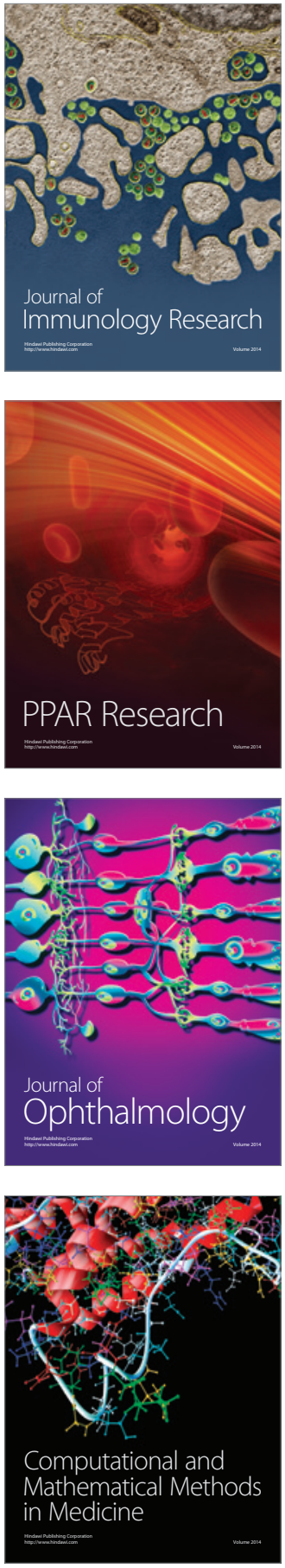

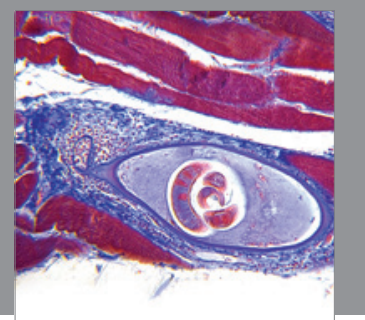

Gastroenterology

Research and Practice
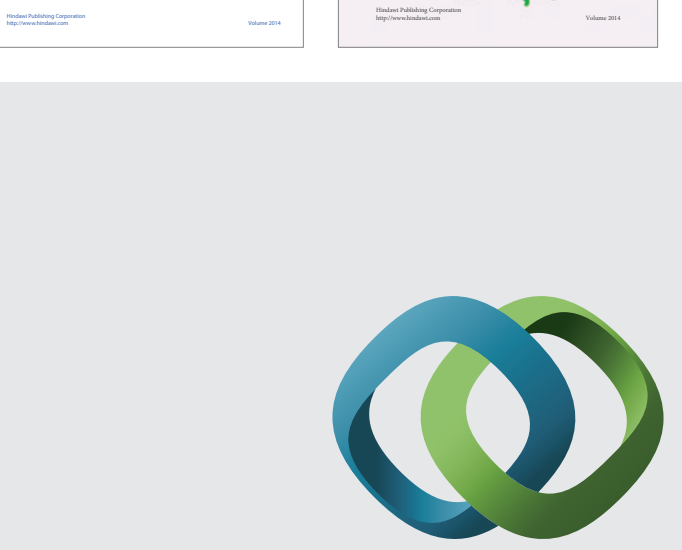

\section{Hindawi}

Submit your manuscripts at

http://www.hindawi.com
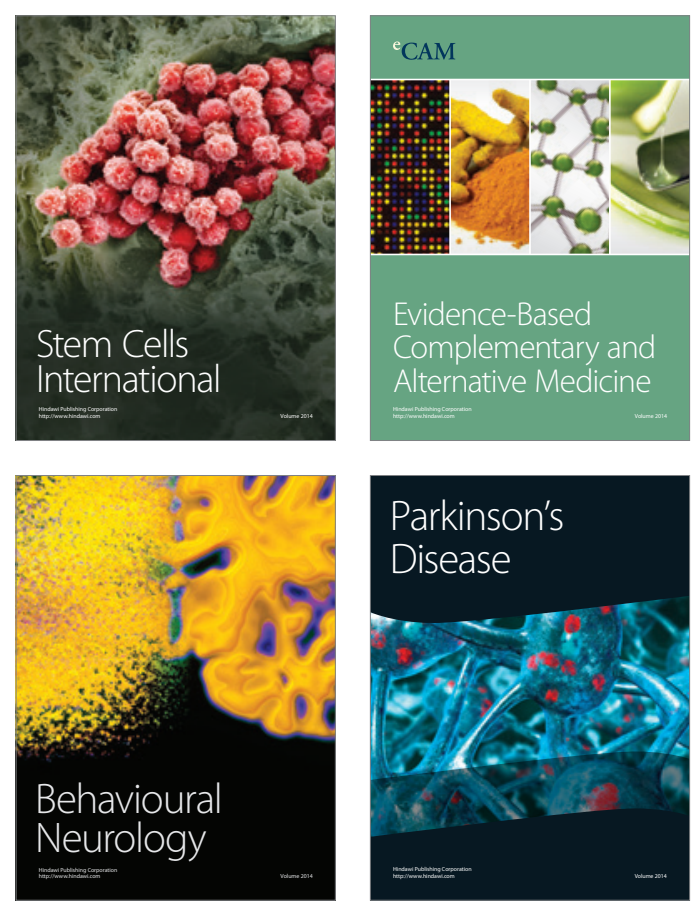

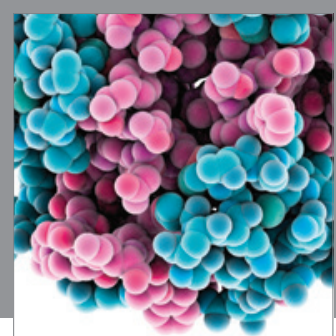

Journal of
Diabetes Research

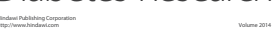

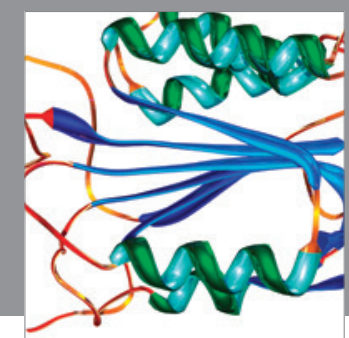

Disease Markers
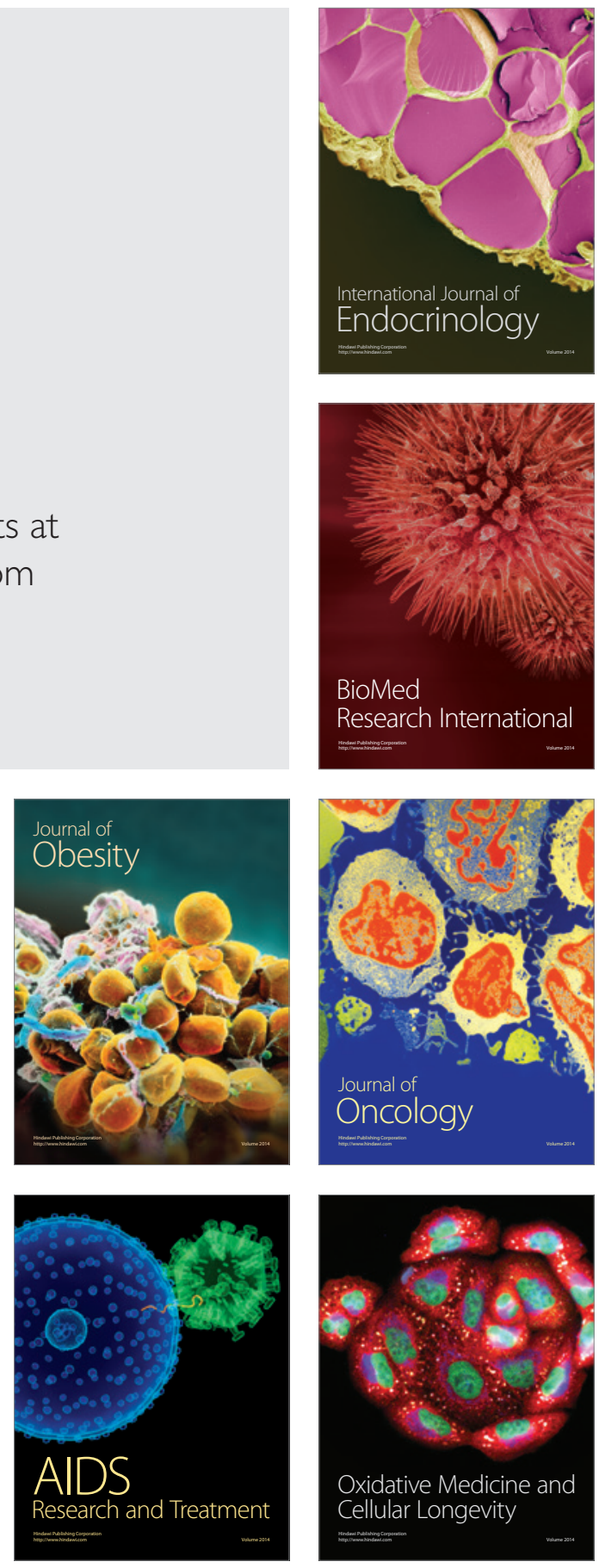